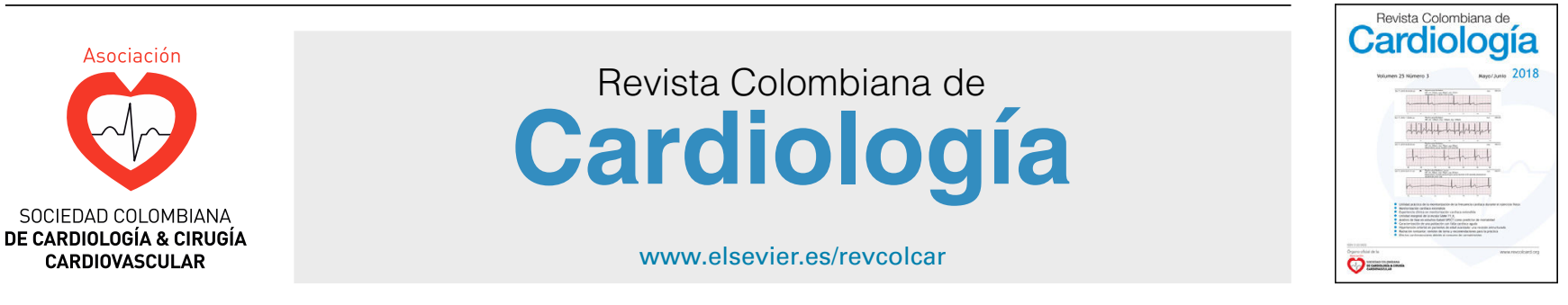

ARTÍCULO ESPECIAL

\title{
Tratamiento de la diabetes en el paciente con insuficiencia cardiaca: Más allá de la hemoglobina glicosilada
}

\section{Gilberto Amed Castillo ${ }^{a, b, *}$ y Enrique Morales-Villegas ${ }^{c}$}

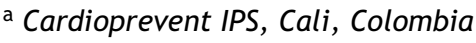

b Centro Médico Imbanaco, Cali, Colombia

c Centro de Investigación Cardiometabólica (CIC), Aguascalientes, México

Recibido el 19 de noviembre de 2019; aceptado el 17 de diciembre de 2019

\section{PALABRAS CLAVE \\ Diabetes; \\ Insuficiencia \\ cardiaca; \\ Tratamiento}

\section{KEYWORDS}

Diabetes;

Heart failure;

Treatment

\begin{abstract}
Resumen
Introducción: los pacientes con diabetes mellitus tipo 2 tienen una prevalencia de insuficiencia cardiaca cuatro veces más alta que la población general.

Objetivo: revisar la evidencia de los diferentes tratamientos antidiabéticos en los pacientes con diabetes mellitus tipo 2 e insuficiencia cardiaca, las consideraciones necesarias cuando hay compromiso de la función renal y los posibles mecanismos que explican los resultados benéficos de estas terapias.

Conclusión: la evidencia actual ha demostrado que algunos de los medicamentos antidiabéticos tienen la capacidad de reducir la tasa de hospitalizaciones por insuficiencia cardiaca, en especial los inhibidores de SGLT2.

(c) 2019 Publicado por Elsevier España, S.L.U. en nombre de Sociedad Colombiana de Cardiología y Cirugía Cardiovascular. Este es un artículo Open Access bajo la licencia CC BY-NC-ND (http: / / creativecommons.org/licenses/by-nc-nd/4.0/).
\end{abstract}

\section{Treatment of diabetes in patients with heart failure: Beyond the glicosilate} hemoglobin

\begin{abstract}
Introduction: The prevalence of heart failure is four times higher in patients with type 2 diabetes mellitus than in the general population.

Objective: To review the evidence of the different diabetic treatments in patients with type 2 diabetes mellitus and heart failure, as well as the considerations that need to be taken into account when kidney function is compromised, and any mechanism that could explain the beneficial results of these therapies.
\end{abstract}

\footnotetext{
* Autor para correspondencia.

Correo electrónico: gcastillob@yahoo.com (G.A. Castillo B).
} 
Conclusion: The current evidence has demonstrated that some diabetic drugs, in particular sodium-glucose cotransporter-2 (SGLT2) inhibitors, can reduce the hospital admission rates due to heart failure.

(c) 2019 Published by Elsevier España, S.L.U. on behalf of Sociedad Colombiana de Cardiología y Cirugía Cardiovascular. This is an open access article under the CC BY-NC-ND license (http:// creativecommons.org/licenses/by-nc-nd/4.0/).

\section{Introducción}

Aunque existe una clara relación entre insuficiencia cardiaca y diabetes mellitus tipo 2, las guías de práctica clínica las han analizado tradicionalmente como entidades aisladas. La prevalencia de insuficiencia cardiaca en los pacientes con diabetes mellitus tipo 2 es cuatro veces más alta que en la población general (entre el 9 y el $22 \%)^{1}$, y es todavía más alta en diabéticos mayores de 60 años $^{2}$. Hoy en día, es claro que la diabetes mellitus tipo 2 es un factor de riesgo para desarrollar insuficiencia cardiaca, pero esta última también es un factor de riesgo para desarrollar diabetes mellitus ${ }^{2}$.

En quienes tienen ambos diagnósticos, insuficiencia cardiaca y diabetes mellitus tipo 2 , hay incremento de la mortalidad, reducción de la calidad de vida y mayores costos de atención ${ }^{3}$. Igualmente, la presencia de diabetes mellitus tipo 2 eleva la mortalidad en aquellos con diagnóstico de insuficiencia cardiaca tanto a nivel intrahospitalario como ambulatorio ${ }^{4,5}$. Otros desenlaces, como hospitalizaciones por insuficiencia cardiaca, readmisiones hospitalarias y calidad de vida, también son más altos en pacientes con diabetes mellitus tipo 2 e insuficiencia cardiaca, que en individuos que tienen sólo insuficiencia cardiaca ${ }^{2}$.

Los pacientes diabéticos sin insuficiencia cardiaca sintomática pueden tener anormalidades subclínicas en la función y en la estructura cardiaca, tales como disfunción sistólica del ventrículo izquierdo, disfunción diastólica, aumento en la masa ventricular izquierda y engrosamiento relativo de la pared ventricular y del tamaño de la aurícula izquierda. Estas anormalidades se asocian con mayor riesgo de insuficiencia cardiaca sintomática y muerte ${ }^{6}$, lo cual implica que las recomendaciones para el tratamiento de los pacientes con diabetes mellitus tipo 2 e insuficiencia cardiaca, también puedan ser útiles en aquellos diabéticos sin insuficiencia cardiaca conocida y con factores de riesgo para desarrollarla en el futuro.

\section{Objetivos glicémicos en pacientes diabéticos con insuficiencia cardiaca}

La hiperglicemia, con o sin diabetes, se asocia con mayor riesgo de desarrollar insuficiencia cardiaca ${ }^{7}$. Los datos disponibles sugieren que el control glicémico intensivo en pacientes con diabetes mellitus tipo 2 establecida no reducen el riesgo de insuficiencia cardiaca ${ }^{8}$. Estudios observacionales afirman que la mortalidad más baja en los pacientes con diabetes mellitus e insuficiencia cardiaca se presenta cuando la HbA1c está entre 7,0 y $8,0 \%{ }^{9}$. Las metas de glicemia deben individualizarse en estos pacientes con el objetivo de reflejar la carga de las comorbilidades, incluida la severidad de la insuficiencia cardiaca y la expectativa de vida del paciente. La meta que se elija debe balancear los beneficios de reducir estrictamente la HbA1c contra riesgos potenciales, como hipoglicemia, polifarmacia y mayores costos. La intensidad del control glicémico debe ir de la mano con el estadio y la severidad de la insuficiencia cardiaca, con una monitorización estrecha de la seguridad y la eficacia de las terapias hipoglicemiantes.

En general se diría que puede buscarse una meta de HbA1c por debajo de 7,0\% en el diabético con insuficiencia cardiaca que no tenga otras complicaciones ni comorbilidades importantes y que cuente con un buen pronóstico de sobrevida a largo plazo $^{2}$. La mayoría de pacientes con diabetes mellitus tipo 2 e insuficiencia cardiaca pueden beneficiarse de tener HbA1c entre 7,0 y $8,0 \%$, y se pudiera considerar una meta entre 8,0 y $8,5 \%$ en los pacientes con una expectativa de vida limitada, insuficiencia cardiaca estadio $D$, enfermedad renal terminal o dependencia de oxígeno ${ }^{2}$.

\section{Elección de la terapia antidiabética adecuada en el paciente con insuficiencia cardiaca}

\section{Metformina}

En la actualidad es la terapia inicial preferida en ausencia de contraindicaciones, dado que es un medicamento efectivo, seguro, bien tolerado y de bajo costo. Hasta 2006 su uso estaba contraindicado en Estados Unidos en pacientes diabéticos con insuficiencia cardiaca debido al riesgo de acidosis láctica. Sin embargo, hoy en día, algunos estudios observacionales sugieren una reducción de la mortalidad total entre los diabéticos que emplean metformina ${ }^{10}$. En conclusión, es razonable emplear metformina en pacientes diabéticos con insuficiencia cardiaca o con riesgo de insuficiencia cardiaca, pero se sugiere que sea suspendida durante condiciones agudas que se asocien con acidosis láctica, como choque cardiogénico o choque distributivo ${ }^{2}$.

\section{Sulfonilúreas}

Aunque existe información contradictoria, en general se puede decir que las sulfonilúreas no se asocian con un incremento en las hospitalizaciones por insuficiencia cardiaca ${ }^{2}$. Si bien el estudio CAROLINA, que comparó directamente la 
aparición de complicaciones cardiovasculares en pacientes que recibían una sulfonilúrea (glimepirida) vs. un inhibidor de DPP-4 (linagliptina), mostró un incremento en la tasa de hipoglicemia en el brazo de glimepirida, no arrojó diferencias respecto a la seguridad cardiovascular ni a la tasa de hospitalización por insuficiencia cardiaca ${ }^{11}$.

\section{Insulina}

Su uso se asocia con aumento de peso, retención de líquidos y mayor riesgo de hipoglicemia, por lo que debería emplearse con moderación en el paciente con insuficiencia cardiaca. Sin embargo, el estudio ORIGIN, que evaluó la seguridad cardiovascular de insulina glargina, no demostró diferencia en ningún desenlace cardiovascular incluyendo las hospitalizaciones por insuficiencia cardiaca aún en el período de tiempo extendido por más de 2,5 años ${ }^{12}$. Por su parte, si bien el estudio DEVOTE, que comparó insulina degludec vs. insulina glargina, demostró menor incidencia de hipoglucemia con insulina degludec, no reveló diferencias respecto a la seguridad cardiovascular entre ambas insulinas $^{13}$.

\section{Tiazolidinedionas}

Los estudios clínicos con estos medicamentos (estudio PROactive con pioglitazona y RECORD con rosiglitazona) han demostrado un incremento en la tasa de hospitalización por insuficiencia cardiaca en pacientes sin insuficiencia cardiaca de base ${ }^{14,15}$. El uso de tiazolidinedionas se asocia con retención de líquidos e incremento en la tasa de hospitalizaciones por insuficiencia cardiaca, aunque sin comprometer la fracción de eyección, lo que sugiere que el mecanismo predominante puede ser la expansión de volumen secundaria a la reabsorción de sodio en el riñón ${ }^{16}$. Por tal motivo, las tiazolidinedionas no se recomiendan en pacientes diabéticos con insuficiencia cardiaca.

\section{Inhibidores de la dipeptidil peptidasa-4 (DPP-4)}

El estudio SAVOR TIMI 53 mostró un incremento significativo del $27 \%$ en la tasa de hospitalizaciones por insuficiencia cardiaca en los pacientes tratados con saxagliptina ${ }^{17}$. Por el contrario, en los estudios EXAMINE ${ }^{18}$ con alogliptina, TECOS $^{19}$ con sitagliptina, CARMELINA ${ }^{20}$ y CAROLINA $^{11}$ con linagliptina no hubo incremento significativo en la tasa de hospitalizaciones por insuficiencia cardiaca. El metaanálisis de Zheng et al. ${ }^{21}$ indicó que los inhibidores de la DPP. 4 se asociaron con $22 \%$ más riesgo de hospitalización por insuficiencia cardiaca en relación con los agonistas de los receptores de GLP-1, y $81 \%$ más riesgo en comparación con los inhibidores de SGLT2. En general, no hay evidencia de que los inhibidores de la DPP-4 provean un beneficio cardiovascular, por lo que no se recomienda su utilización como primera o segunda línea terapéutica, específicamente en los pacientes diabéticos con insuficiencia cardiaca o alto riesgo de desarrollarla².

\section{Agonistas de los receptores de GLP-1}

Los análogos de GLP1, liraglutida ${ }^{22}$, semaglutida ${ }^{23}$, albiglutida $^{24}$ y dulaglutida ${ }^{25}$, han demostrado beneficio cardiovascular al reducir el desenlace cardiovascular combinado 3-P MACE (muerte cardiovascular, infarto de miocardio o ataque cerebrovascular no fatal), pero no modifican la tasa de hospitalizaciones por insuficiencia cardiaca. Algunos modelos en animales sugieren que la administración de un agonista de los receptores de GLP-1 puede mejorar la contractilidad del ventrículo izquierdo, reducir la hipertrofia, atenuar el estrés oxidativo y mejorar la sobrevida ${ }^{26,27}$. Por su parte, el estudio FIGHT, que incluyó 300 pacientes con insuficiencia cardiaca con fracción de eyección reducida y hospitalización reciente por insuficiencia cardiaca y los aleatorizó a recibir liraglutida $1,8 \mathrm{mg} / \mathrm{d}$ vs. placebo por seis meses, no encontró diferencias en la estabilidad clínica poshospitalización ni en las readmisiones por insuficiencia cardiaca ${ }^{28}$. En general, los agonistas de los receptores de GLP-1 mencionados se consideran eficaces en la reducción del desenlace cardiovascular combinado 3-P MACE, mas no benéficos en la reducción de hospitalizaciones por insuficiencia cardiaca. En pacientes con diabetes mellitus e insuficiencia cardiaca con fracción de eyección reducida ya conocida y descompensación reciente, los agonistas de los receptores de GLP-1 deberían ser utilizados con precaución ${ }^{2}$.

\section{Inhibidores SGLT2}

Tienen múltiples efectos asociados a su beneficio cardiorrenal, entre ellos su efecto glucosúrico y natriurético que, además de reducir el volumen intravascular, son determinantes de la activación del denominado "feedback" túbulo-glomerular con vasoconstricción de la arteriola aferente y reducción de la presión intraglomerular. El estudio EMPAREG-OUTCOME con empagliflozina en dosis de $10 \mathrm{y}$ de $25 \mathrm{mg}$ al día, sorprendió tanto por la reducción en la tasa de hospitalizaciones por insuficiencia cardiaca en un $35 \%$, como por la clara diferenciación de las curvas a favor del brazo de empagliflozina desde las primeras semanas de tratamiento ${ }^{29}$. Los estudios del programa CANVAS mostraron también una reducción del $33 \%$ en la tasa de hospitalizaciones por insuficiencia cardiaca en los pacientes que recibieron canagliflozina en comparación con placebo ${ }^{30}$. Análisis posteriores del CANVAS sugieren que se obtienen mayores beneficios sobre la morbimortalidad en aquellos pacientes diabéticos con insuficiencia cardiaca previa ${ }^{31}$. Finalmente, el estudio DECLARE-TIMI 58, que incluyó un porcentaje mucho más alto de pacientes diabéticos de alto riesgo pero sin enfermedad cardiovascular establecida $(59,4 \%$ en DECLARE vs. $34.4 \%$ en CANVAS vs. $0 \%$ en EMPAREG-OUTCOME), encontró que los pacientes tratados con dapagliflozina $10 \mathrm{mg} / \mathrm{d}$ tuvieron una reducción en la tasa de hospitalizaciones por insuficiencia cardiaca del $27 \%^{32}$. Zelniker et al. publicaron un metaanálisis y revisión sistemática de los estudios de desenlace cardiovascular de los inhibidores de SGLT2 en prevención primaria y secundaria. En este metaanálisis, los inhibidores de SGLT2 se asociaron con una reducción del desenlace combinado de hospitalización por insuficiencia cardiaca o muerte de causa 
cardiovascular mayor del $20 \%$, en los pacientes diabéticos con o sin historia de insuficiencia cardiaca ${ }^{33}$.

Los resultados de todos los inhibidores SGLT2 motivaron la realización de estudios específicos en pacientes con diagnóstico ya confirmado de insuficiencia cardiaca con fracción de eyección reducida y preservada, independientemente de la presencia o ausencia de diabetes mellitus tipo 2. El estudio DAPA-HF evaluó 4.744 pacientes con insuficiencia cardiaca con fracción de eyección $\leq 40 \%$, en clase funcional II a IV y niveles elevados del fragmento $\mathrm{N}$ terminal del pro-BNP (NT-proBNP), y los aleatorizó a recibir dapagliflozina $10 \mathrm{mg} / \mathrm{d}$ vs. placebo. Todos los pacientes recibieron terapia óptima para el manejo de la insuficiencia cardiaca incluyendo un inhibidor del sistema renina-angiotensina-aldosterona (> 90\% del total de participantes), betabloqueadores (más del 96\%) y antagonistas de los receptores de mineralocorticoides (más del 70\%). Los pacientes tratados con dapagliflozina presentaron una reducción estadísticamente significativa del $26 \%$ en el desenlace combinado primario (muerte de causa cardiovascular y empeoramiento de la insuficiencia cardiaca documentado por hospitalización o visita al servicio de urgencias). La muerte cardiovascular fue $18 \%$ menor en el brazo dapagliflozina ${ }^{34}$.

Patorno et al. publicaron el primer análisis interino del estudio EMPRISE, en el que evaluaron el riesgo de hospitalizaciones por insuficiencia cardiaca en 16.443 diabéticos tipo 2 que habían iniciado terapia con empagliflozina vs. sitagliptina a partir de agosto de 2014. Dicho análisis mostró que los pacientes que habían iniciado empagliflozina tuvieron $50 \%$ menos hospitalizaciones por insuficiencia cardiaca en comparación con aquellos que habían iniciado sitagliptina en un período de seguimiento promedio de 5,3 meses e independientemente de presentar enfermedad cardiovascular preexistente o no no, $^{35,36}$.

En resumen, los inhibidores SGLT2 son la primera clase de medicamentos antidiabéticos que han demostrado reducir el riesgo de hospitalización por insuficiencia cardiaca en pacientes con diabetes mellitus tipo 2 . Los análisis secundarios de los estudios de seguridad cardiovascular sugieren que los inhibidores de SGLT2 reducen el riesgo de hospitalización por insuficiencia cardiaca tanto en los pacientes que ya tienen diagnóstico conocido de insuficiencia cardiaca como en aquellos que no. Por lo tanto, los inhibidores de SGLT2 deben ser los medicamentos de primera línea en diabéticos con insuficiencia cardiaca ya conocida y en diabéticos con alto riesgo de desarrollar insuficiencia cardiaca ${ }^{2}$. Hasta el momento, dapagliflozina ha demostrado ser efectiva en pacientes con insuficiencia cardiaca y fracción de eyección reducida, incluso en pacientes sin diabetes mellitus tipo 2 . Están en curso varios estudios, como el EMPEROR-REDUCED, que pretende evaluar la eficacia de empagliflozina en pacientes con insuficiencia cardiaca y fracción de eyección reducida (no limitado a pacientes con diabetes mellitus tipo 2), y el SOLOIST-WHF, que evaluará los efectos de sotagliflozina en diabéticos tipo 2 que hayan presentado un deterioro de la insuficiencia cardiaca recientemente.

En el campo de la insuficiencia cardiaca con fracción de eyección preservada se esperan los resultados del EMPEROR-PRESERVED (con empagliflozina) y del DELIVER (con dapagliflozina). Hasta el momento no hay evidencia para afirmar que estos resultados tan favorables con el uso de inhibidores de SGLT2 en diabéticos tipo 2 con insuficiencia cardiaca y fracción de eyección reducida, sea igual en aquellos que tienen insuficiencia cardiaca con fracción de eyección preservada.

\section{Tratamiento del paciente diabético tipo 2 con insuficiencia cardiaca y enfermedad renal crónica}

A pesar de la alta prevalencia de enfermedad renal crónica tanto en los pacientes con diabetes mellitus tipo 2 como en los pacientes con insuficiencia cardiaca, hay datos limitados acerca del mejor tratamiento para aquellos en quienes coincidan las tres entidades.

Los inhibidores de SGLT2 son los antidiabéticos predilectos en el paciente con diabetes mellitus tipo 2 e insuficiencia cardiaca con fracción de eyección reducida y función renal normal, pero su uso en pacientes con enfermedad renal crónica puede verse limitado, especialmente por aspectos de seguridad y eficacia en el control glucémico con tasa de filtración glomerular menor a $45 \mathrm{ml} / \mathrm{min} / 1,73 \mathrm{~m}^{2}$.

Por otra parte, el estudio EMPAREG-OUTCOME, al igual que el estudio CANVAS $^{30}$, excluyeron pacientes con tasa de filtración glomerular por debajo de $30 \mathrm{ml} / \mathrm{min}^{29}$. El DECLARE-TIMI 58 tenía como criterio de inclusión tener una tasa de filtración glomerular $\geq 60 \mathrm{ml} / \mathrm{min}^{32}$, pero el DAPA-HF incluyó pacientes con tasa de filtración glomerular $\geq 30 \mathrm{ml} / \mathrm{min}^{34}$.

Los estudios de seguridad cardiovascular en pacientes con función renal comprometida aún no se han completado, a excepción del CREDENCE con canagliflozina, que incluyó pacientes con tasa de filtración glomerular $\geq 30 \mathrm{ml} / \mathrm{min}$ y que se detuvo prematuramente por los hallazgos a favor de canagliflozina. En este estudio el riesgo de hospitalización por insuficiencia cardiaca se redujo en un 39\%. Hay dos estudios en curso: el EMPA-KIDNEY con empagliflozina, que incluye pacientes con tasa de filtración glomerular $\geq$ $20 \mathrm{ml} / \mathrm{min}$, y el DAPA-CKD con dapagliflozina, que incluye pacientes con tasa de filtración glomerular $\geq 25 \mathrm{ml} / \mathrm{min}$. Sin embargo, no se cuenta aún con los resultados para conocer si con ese grado de disfunción renal siguen siendo medicamentos eficaces y seguros para reducir las hospitalizaciones por insuficiencia cardiaca.

En conclusión, en los pacientes diabéticos con insuficiencia cardiaca con fracción de eyección reducida y enfermedad renal crónica con tasa de filtración glomerular por encima de $30 \mathrm{ml} / \mathrm{min}$ puede emplearse metformina (dosis ajustada según el grado de disfunción renal) y un inhibidor de SGLT2.

Con una tasa de filtración glomerular por debajo de $30 \mathrm{ml} / \mathrm{min}$ la metformina está contraindicada y los inhibidores de SGLT2 no tienen hasta el momento suficiente evidencia. Con los datos del EMPA-KIDNEY y DAPA-CKD es posible que ese umbral de la tasa de filtración glomerular se disminuya hasta $25 \mathrm{o}$, incluso, $20 \mathrm{ml} / \mathrm{min}$.

\section{¿Por qué los inhibidores de SGLT2 reducen las hospitalizaciones por insuficiencia cardiaca?}

Se han propuesto varias hipótesis para explicar los mecanismos de protección cardiaca con los inhibidores de SGLT2. 
A continuación se analizan las tres hipótesis que parecen tener más fuerza: la mejoría en el desempeño del ventrículo izquierdo secundaria a la disminución de la precarga y la poscarga, el cambio en el sustrato metabólico por parte de los cardiomiocitos y, finalmente, el bloqueo del intercambiador de $\mathrm{Na}^{+} / \mathrm{H}^{+}$en el miocardio.

Uno de los hallazgos más interesantes observados en el EMPAREG-OUTCOME, es la separación de las curvas desde antes de los primeros seis meses para el desenlace primario combinado y para la muerte de causa cardiovascular. Para el desenlace de hospitalizaciones por insuficiencia cardiaca, las curvas se separan desde el primer mes de tratamiento ${ }^{29}$, lo cual contrasta con lo observado con medicamentos que alteran la fisiopatología de la placa aterosclerótica, como por ejemplo las estatinas, los cuales típicamente muestran beneficio en la mortalidad después del primer año de exposición. Esta rapidez del efecto de los inhibidores de SGLT2 sugiere que los beneficios cardiovasculares suceden, al menos en parte, por mecanismos hemodinámicos. El uso de inhibidores de SGLT2 ocasiona diuresis osmótica y natriuresis, las cuales causan disminución del volumen intravascular circulante, así como reducción de la presión arterial y de la rigidez arterial; esto lleva a disminución de la precarga y de la postcarga, mejorando el funcionamiento del ventrículo izquierdo ${ }^{37}$. Sin embargo, otros diuréticos, como las tiazidas y los de asa, pudieran producir un efecto similar, pero no han demostrado reducción de desenlaces cardiovasculares como los inhibidores de SGLT2. El factor diferenciador más importante puede ser la capacidad de estos últimos para reducir el volumen intersticial selectivamente en una proporción mayor, que para reducir el volumen intravascular ${ }^{38,39}$.

El siguiente mecanismo postulado tiene que ver con el cambio del sustrato energético por uno más eficiente, de modo que mejore el metabolismo cardiaco. La disminución en la producción de insulina y el incremento en la síntesis de glucagón que se observan al administrar un inhibidor de SGLT2, llevan a un incremento en la producción de cuerpos cetónicos hepáticos, especialmente $\beta$-hidroxibutirato. Este constituye un supercombustible para el cardiomiocito, el cual pasa a utilizar los cuerpos cetónicos como sustrato energético en lugar de los ácidos grasos libres. Al cambiar de sustrato energético se reduce la demanda miocárdica de oxígeno y se mejora la función cardiaca y renal ${ }^{40,41}$. Además, es posible que el $\beta$-hidroxibutirato tenga propiedades antioxidantes y antiarrítmicas ${ }^{42}$. También hay evidencia que indica que los inhibidores de SGLT2 aumentan la síntesis de eritropoyetina llevando a un incremento en la masa total de glóbulos rojos ${ }^{43}$. Al recuperarse la eficiencia energética del cardiomiocito y tener mayor masa de glóbulos rojos, el aporte de oxígeno al miocardio mejoraría significativamente.

Finalmente, otra de las hipótesis más recientes sugiere que los inhibidores de SGLT2 pueden inhibir directamente la isoforma $1 \mathrm{del}$ intercambiador de $\mathrm{Na}^{+} / \mathrm{H}^{+}$en el miocardio. $\mathrm{El}$ incremento en la actividad del intercambiador $\mathrm{Na}^{+} / \mathrm{H}^{+}$se ha identificado como un marcador temprano de daño del cardiomiocito, falla cardiaca y muerte cardiovascular ${ }^{38,39}$. El empleo de un inhibidor de SGLT2 bloquea dicho intercambiador reduciendo los niveles de sodio y calcio en el citoplasma del cardiomiocito, a merced del incremento de los niveles de calcio al interior de la mitocondria ${ }^{44}$.
Con la publicación del DAPA-HF, se sugiere que todos aquellos mecanismos que se consideraban probables como explicación de los efectos benéficos de los inhibidores de SGLT2, generan ahora más dudas por los hallazgos observados en pacientes sin diabetes. Adicionalmente, se plantea que los beneficios de los inhibidores de SGLT2 en la falla cardíaca probablemente estén relacionados con una acción de estos medicamentos para promover mecanismos de viabilidad de los cardiomiocitos y mejorar las vías que conducen a la muerte de los mismos ${ }^{45}$.

\section{La importancia de un equipo interdisciplinario para tratar al diabético con insuficiencia cardiaca}

El tratamiento de los pacientes diabéticos tipo 2 que presentan, además, insuficiencia cardiaca puede llegar a ser muy complejo, pues requiere de medidas de autocuidado que difieren para cada una de las enfermedades. Los ajustes en la dieta, el consumo de líquidos, la actividad física, el control del peso y el manejo del estrés pueden ser difíciles de tratar si el paciente escucha recomendaciones distintas realizadas por los profesionales que lo atienden. En un pequeño metaanálisis cualitativo, los pacientes con diabetes mellitus tipo 2 e insuficiencia cardiaca reportaron falta de conocimiento, habilidad y eficacia para integrar las múltiples recomendaciones de autocuidado, lo que los llevó a priorizar unos sobre otros (por ejemplo, monitorizaban sus niveles de glucosa diariamente pero no su peso corporal) ${ }^{46}$. En un registro norteamericano que incluyó 1.901 pacientes diabéticos se encontró que tener insuficiencia cardiaca severa se asociaba con menor priorización de la diabetes y menores puntajes en las escalas de autocuidado ${ }^{47}$. Por otro lado, tener múltiples profesionales atendiendo al mismo paciente puede generar información contradictoria que confunde al enfermo y a su familia. Además, dado que la insuficiencia cardiaca tiende a ser más sintomática, el autocuidado para la diabetes mellitus tipo 2 pasa a ser considerado menos importante y, por tanto, se afecta el control metabólico ${ }^{2}$. Es importante que los diferentes prestadores de salud estén de acuerdo con las mismas recomendaciones respecto al autocuidado, y a establecer prioridades cuando la capacidad es limitada.

Construir un equipo básico de atención, que incluya médicos generales y especialistas en medicina interna, endocrinología y cardiología, así como enfermeras, nutricionistas y trabajadores sociales, puede ayudar a alcanzar mejores resultados clínicos. La premisa de un trabajo basado en el equipo interdisciplinario de atención para toda enfermedad crónica es desarrollar programas de trabajo individualizados considerando la preferencia de los pacientes y la coordinación efectiva del cuidado a través de todos los miembros del equipo de salud ${ }^{2}$.

La implementación de un programa individualizado de actividad física puede ayudar a mejorar los desenlaces tanto de la diabetes mellitus tipo 2 como de la insuficiencia cardiaca. En el estudio HF-ACTION, 2.331 individuos con insuficiencia cardiaca con fracción de eyección reducida fueron aleatorizados a un programa de entrenamiento físico o a manejo médico óptimo. Un $32 \%$ de todos los individuos que ingresaron al estudio eran diabéticos, y en ellos se 
evidenció menor adherencia al entrenamiento físico. Pese a ello, los pacientes diabéticos aleatorizados al ejercicio mostraron mejoría significativa en el consumo pico de oxígeno y en la distancia recorrida en la caminata de 6 minutos, en comparación con los pacientes en terapia convencional ${ }^{48}$. Por tal motivo es muy importante que los pacientes con insuficiencia cardiaca y diabetes mellitus tipo 2 sean remitidos a un programa de rehabilitación cardiaca y se les insista en la importancia de la adherencia a las recomendaciones sobre actividad física.

\section{Conclusiones}

La prevalencia de insuficiencia cardiaca entre los pacientes diabéticos es cuatro veces más alta que en la población general, aunque un porcentaje elevado de diabéticos puede presentar alteraciones cardiacas estructurales y funcionales antes de aparecer insuficiencia cardiaca clínicamente manifiesta. Estos pacientes tienen mayor riesgo de mortalidad y de desarrollar en el futuro insuficiencia cardiaca sintomática. Los inhibidores de SGLT2 (empagliflozina, canagliflozina y dapagliflozina) han demostrado ser eficaces en la reducción de las hospitalizaciones por insuficiencia cardiaca entre los pacientes diabéticos, tanto en aquellos con insuficiencia cardiaca conocida como en aquellos sin diagnóstico previo de esta entidad. Por tal razón, los inhibidores de SGLT2, junto con la metformina, son los medicamentos de primera línea en el diabético con insuficiencia cardiaca. Sin embargo, estos no se han evaluado aún en pacientes con tasa de filtración glomerular por debajo de $30 \mathrm{ml} / \mathrm{min}$.

Recientemente, el estudio DAPA-HF demostró la eficacia de dapagliflozina en pacientes con diagnóstico previo confirmado de insuficiencia cardiaca con fracción de eyección reducida, con y sin diabetes. Los hallazgos observados en los pacientes no diabéticos han hecho revaluar aquellas hipótesis sobre los mecanismos fisiológicos que intentaban explicar las razones de los resultados clínicos favorables de los inhibidores de SGLT2.

Es probable que en el futuro los inhibidores de SGLT2 empiecen a estudiarse en otro tipo de enfermedades diferentes a la diabetes mellitus tipo 2 puesto que, posiblemente, la reducción de la glucosa sea solo uno de sus efectos farmacológicos. Hoy en día se conoce que estos medicamentos ejercen diferentes efectos incluso en tejidos en los que no se han evidenciado receptores SGLT2. Este hecho abre el panorama a mecanismos de acción indirectos que aún se desconocen en su totalidad.

\section{Conflicto de intereses}

Gilberto Castillo ha recibido honorarios como conferencista de Boehringer-Ingelheim, AstraZeneca, NovoNordisk, Sanofi, Pfizer, Bayer y Abbott.

Enrique Morales ha recibido honorarios como conferencista, investigador y consultor por los siguientes consorcios farmacéuticos: Abbott, Amgen, BoeheringerIngelheim, Bristol Myers/Astra Zeneca, Janssen-Cilag, Kowa, Eli Lilly, Merck Sharp and Dohme, Novartis, Novo Nordisk, Pfizer, Roche, Sanofi, Servier, Takeda y Theracos.

\section{Bibliografía}

1. Nichols GA, Gullion CM, Koro CE, Ephross SA, Brown JB. The incidence of congestive heart failure in type 2 diabetes: an update. Diabetes Care. 2004;27:1879-84.

2. Dunlay SM, Givertz MM, Aguilar D, Allen LA, Chan M, Desai AS, et al. Type 2 diabetes mellitus and heart failure: a scientific statement from the American Heart Association and the Heart Failure Society of America. Circulation. 2019;140:e294-324.

3. Vardeny 0 . The sweet spot: heart failure prevention with SGLT2 inhibitors. Am J Med. 2019;piiS0002-9343:30706-15, http:// dx.doi.org/10.1016/j.amjmed.2019.08.013 [Epub ahead of print].

4. Targher G, Dauriz M, Laroche C, Temporelli PL, Hassanein M, Seferovic PM, et al. In-hospital and 1-year mortality associated with diabetes in patients with acute heart failure: results from the ESC-HFA Heart Failure Long-Term Registry. Eur J Heart Fail. 2017; 19:54-65.

5. Dauriz M, Targher G, Laroche C, Temporelli PL, Ferrari R, Anker $\mathrm{S}$, et al. Association between diabetes and 1-year adverse clinical outcomes in a multinational cohort of ambulatory patients with chronic heart failure: results from the ESC-HFA Heart Failure Long-Term Registry. Diabetes Care. 2017;40:671-8.

6. Swoboda PP, McDiarmid AK, Erhayiem B, Ripley DP, Dobson LE, Garg P, et al. Diabetes mellitus, microalbuminuria, and subclinical cardiac disease: identification and monitoring of individuals at risk of heart failure. J Am Heart Assoc. 2017;6:e005539, doi: 10.1161/JAHA.117.005539.

7. Pazin-Filho A, Kottgen A, Bertoni AG, Russell SD, Selvin E, Rosamond WD, et al. HbA $1 \mathrm{c}$ as a risk factor for heart failure in persons with diabetes: the Atherosclerosis Risk in Communities (ARIC) study. Diabetologia. 2008;51:2197-204.

8. Castagno D, Baird-Gunning J, Jhund PS, Biondi-Zoccai G, MacDonald MR, Petrie MC, et al. Intensive glycemic control has no impact on the risk of heart failure in type 2 diabetic patients: evidence from a 37,229 patient meta-analysis. Am Heart J. 2011;162:938-48, e2.

9. Elder DH, Singh JS, Levin D, Donnelly LA, Choy AM, George $J$, et al. Mean HbA1c and mortality in diabetic individuals with heart failure: a population cohort study. Eur J Heart Fail. 2016;18:94-102.

10. Shah DD, Fonarow GC, Horwich TB. Metformin therapy and outcomes in patients with advanced systolic heart failure and diabetes. J Card Fail. 2010;16:200-6.

11. Rosenstock J, Kahn SE, Johansen OE, Zinman B, Espeland MA, Woerle HJ, et al. Effect of Linagliptin vs Glimepiride on Major Adverse Cardiovascular Outcomes in Patients With Type 2 Diabetes: The CAROLINA Randomized Clinical Trial. JAMA. 2019, http://dx.doi.org/10.1001/jama.2019.13772 [Epub ahead of print].

12. ORIGIN Trial Investigators. Cardiovascular and other outcomes postintervention with insulin glargine and omega-3 fatty acids (ORIGINALE). Diabetes Care. 2016;39:709-16.

13. Marso SP, McGuire DK, Zinman B, Poulter NR, Emerson SS, Pieber TR, et al. Efficacy and safety of degludec versus glargine in type 2 diabetes. N Engl J Med. 2017;377:723-32.

14. Dormandy JA, Charbonnel B, Eckland DJ, Erdmann E, MassiBenedetti M, Moules IK, et al. Secondary prevention of macrovascular events in patients with type 2 diabetes in the PROactive Study (PROspective pioglitAzone Clinical Trial In macroVascular Events): a randomised controlled trial. Lancet. 2005;366:1279-89.

15. Home PD, Pocock SJ, Beck-Nielsen H, Curtis PS, Gomis R, Hanefeld $M$, et al. Rosiglitazone evaluated for cardiovascular outcomes in oral agent combination therapy for type 2 diabetes (RECORD): a multicentre, randomised, open-label trial. Lancet. 2009;373:2125-35. 
16. Guan Y, Hao C, Cha DR, Rao R, Lu W, Kohan DE, et al. Thiazolidinediones expand body fluid volume through PPARgamma stimulation of ENaC-mediated renal salt absorption. Nat Med. 2005;11:861-6.

17. Scirica BM, Braunwald E, Raz I, Cavender MA, Morrow DA, Jarolim P, et al. Heart failure, saxagliptin, and diabetes mellitus: observations from the SAVOR-TIMI 53 randomized trial. Circulation. 2014;130:1579-88.

18. White WB, Cannon CP, Heller SR, Nissen SE, Bergenstal RM, Bakris GL, et al. Alogliptin after acute coronary syndrome in patients with type 2 diabetes. N Engl J Med. 2013;369: 1327-35.

19. McGuire DK, Van de Werf F, Armstrong PW, Standl E, Koglin J, Green JB, et al. Association between sitagliptin use and heart failure hospitalization and related outcomes in type 2 diabetes mellitus: secondary analysis of a randomized clinical trial. JAMA Cardiol. 2016;1:126-35.

20. McGuire DK, Alexander JH, Johansen OE, Perkovic V, Rosenstock $J$, Cooper ME, et al. Linagliptin Effects on Heart Failure and Related Outcomes in Individuals With Type 2 Diabetes Mellitus at High Cardiovascular and Renal Risk in CARMELINA. Circulation. 2019;139:351-61.

21. Zheng SL, Roddick AJ, Aghar-Jaffar R, Shun-Shin MJ, Francis D, Oliver N, et al. Association between use of sodium-glucose cotransporter 2 inhibitors, glucagon-like peptide 1 agonists, and dipeptidyl peptidase 4 inhibitors with all-cause mortality in patients with type 2 diabetes: a systematic review and metaanalysis. JAMA. 2018;319:1580-91.

22. Marso SP, Daniels GH, Brown-Frandsen K, Kristensen P, Mann JF, Nauck MA, et al. Liraglutide and cardiovascular outcomes in type 2 diabetes. N Engl J Med. 2016;375:311-22.

23. Marso SP, Bain SC, Consoli A, Eliaschewitz FG, Jódar E, Leiter LA, et al. Semaglutide and cardiovascular outcomes in patients with type 2 diabetes. N Engl J Med. 2016;375:1834-44.

24. Hernandez AF, Green JB, Janmohamed S, D'Agostino RB $\mathrm{Sr}$, Granger CB, Jones NP, et al. Albiglutide and cardiovascular outcomes in patients with type 2 diabetes and cardiovascular disease (Harmony Outcomes): a double-blind, randomised placebo-controlled trial. Lancet. 2018;392(10157): 1519-29.

25. Gerstein HC, Colhoun HM, Dagenais GR, Diaz R, Lakshmanan $\mathrm{M}$, Pais $\mathrm{P}$, et al. Dulaglutide and cardiovascular outcomes in type 2 diabetes (REWIND): a double-blind, randomised placebocontrolled trial. Lancet. 2019;394(10193):121-30.

26. Nikolaidis LA, Elahi D, Hentosz T, Doverspike A, Huerbin R, Zourelias L, et al. Recombinant glucagon-like peptide-1 increases myocardial glucose uptake and improves left ventricular performance in conscious dogs with pacing-induced dilated cardiomyopathy. Circulation. 2004;110:955-61.

27. Monji A, Mitsui T, Bando YK, Aoyama M, Shigeta T, Murohara T. Glucagon-like peptide-1 receptor activation reverses cardiac remodeling via normalizing cardiac steatosis and oxidative stress in type 2 diabetes. Am J Physiol Heart Circ Physiol. 2013;305:H295-304.

28. Margulies KB, Hernandez AF, Redfield MM, Givertz MM, Oliveira GH, Cole R, et al. Effects of liraglutide on clinical stability among patients with advanced heart failure and reduced ejection fraction: a randomized clinical trial. JAMA. 2016;316:500-8.

29. Zinman B, Wanner C, Lachin JM, Fitchett D, Bluhmki E, Hantel $\mathrm{S}$, et al. Empagliflozin, cardiovascular outcomes, and mortality in type 2 diabetes. N Engl J Med. 2015;373:2117-28.

30. Neal B, Perkovic V, Mahaffey KW, de Zeeuw D, Fulcher G, Erondu $\mathrm{N}$, et al. Canagliflozin and cardiovascular and renal events in type 2 diabetes. N Engl J Med. 2017;377:644-57.
31. Rådholm K, Figtree G, Perkovic V, Solomon SD, Mahaffey KW, de Zeeuw D, et al. Canagliflozin and heart failure in type 2 diabetes mellitus. Circulation. 2018;138:458-68.

32. Wiviott SD, Raz I, Bonaca MP, Mosenzon O, Kato ET, Cahn A, et al. Dapagliflozin and cardiovascular outcomes in type 2 diabetes. N Engl J Med. 2019;380:347-57.

33. Zelniker TA, Wiviott SD, Raz I, Im K, Goodrich EL, Bonaca MP, et al. SGLT2 inhibitors for primary and secondary prevention of cardiovascular and renal outcomes in type 2 diabetes: a systematic review and meta-analysis of cardiovascular outcome trials. Lancet. 2019;393(10166):31-9.

34. McMurray JJV, Solomon SD, Inzucchi SE, Køber L, Kosiborod MN, Martínez FA. Dapagliflozin in patients with heart failure and reduced ejection fraction. N Engl J Med. 2019, http://dx.doi.org/10.1056/NEJMoa1911303 [Epub ahead of print].

35. Patorno E, Pawar A, Franklin JM, Najafzadeh M, DéruazLuyet A, Brodovicz KG. Empagliflozin and the risk of heart failure hospitalization in routine clinical care. Circulation. 2019;139:2822-30.

36. Perkovic V, Jardine MJ, Neal B, Bompoint S, Heerspink HJL. Charytan diabetes mellitus, et al. Canagliflozin and renal outcomes in type 2 diabetes and nephropathy. N Engl J Med. 2019;380:2295-306.

37. Custodio JS Jr, Duraes AR, Abreu M, Albuquerque Rocha N, Roever L. SGLT2 inhibition and heart failure-current concepts. Heart Fail Rev. 2018;23:409-18.

38. Verma S, McMurray JJV. SGLT2 inhibitors and mechanisms of cardiovascular benefit: a state-of-the-art review. Diabetologia. 2018;61:2108-17.

39. Wojcik C, Warden BA. Mechanisms and evidence for heart failure benefits from SGLT2 Inhibitors. Curr Cardiol Rep. 2019;2:130.

40. Ferrannini E, Mark M, Mayoux E. CV protection in the EMPAREG OUTCOME trial: a "thrifty substrate" hypothesis. Diabetes Care. 2016;39:1108-14.

41. Mudaliar S, Alloju S, Henry RR. Can a shift in fuel energetics explain the beneficial cardiorenal outcomes in the EMPAREG OUTCOME study? A unifying hypothesis. Diabetes Care. 2016;39:1115-22.

42. Cotter DG, Schugar RC, Crawford PA. Ketone body metabolism and cardiovascular disease. Am J Phys Heart Circ Phys. 2013;304:H1060-76.

43. Sano M, Takei M, Shiraishi Y, Suzuki Y. Increased hematocrit during sodium-glucose cotransporter 2 inhibitor therapy indicates recovery of tubulointerstitial function in diabetic kidneys. J Clin Med Res. 2016;8:844-7.

44. Packer M, Anker SD, Butler J, Filippatos G, Zannad F. Effects of sodium-glucose cotransporter 2 inhibitors for the treatment of patients with heart failure: proposal of a novel mechanism of action. JAMA Cardiol. 2017;2:1025-9.

45. Packer M. Lessons learned from the DAPA-HF trial concerning the mechanisms of benefit of SGLT2 inhibitors on heart failure events in the context of other large-scale trials nearing completion. Cardiovasc Diabetol. 2019;18:129.

46. Dickson VV, Buck H, Riegel B. A qualitative meta-analysis of heart failure self-care practices among individuals with multiple comorbid conditions. J Card Fail. 2011;17:413-9.

47. Kerr EA, Heisler M, Krein SL, Kabeto M, Langa KM, Weir D, et al. Beyond comorbidity counts: how do comorbidity type and severity influence diabetes patients' treatment priorities and self-management? J Gen Intern Med. 2007;22:1635-40.

48. Banks AZ, Mentz RJ, Stebbins A, Mikus CR, Schulte PJ, Fleg JL, et al. Response to exercise training and outcomes in patients with heart failure and diabetes mellitus: insights from the HFACTION Trial. J Card Fail. 2016;22:485-91. 